\title{
Delirio de Clerambault: entre un deseo de amor irreductible y la atenuación de un delirio erotomaníaco. Respuesta clínica con neuroléptico de liberación prolongada. A propósito de un caso.
}

Clerambault's delusion: between a desire of uncompromising love and the attenuation of a erotomanic delusion. Clinical response with neuroleptic of long liberation. A case report.

$M^{\mathrm{a}}$ Guadalupe Sorribes Molina a ${ }^{\mathrm{a}}$, Alfonso Pedrós Roseló ${ }^{\mathrm{b}}$, Miguel Barberán Navalón a , Pedro Martínez Mollá ${ }^{\text {b. }}$

${ }^{a}$ Residente en Psiquiatría. ${ }^{b}$ Psiquiatra. ${ }^{a, b, c, d}$ Servicio de Psiquiatría. Hospital Lluís Alcanyís. Xátiva. Valencia, España.

Correspondencia: Alfonso Pedrós Roselló(pedros_alf@gva.es)

Recibido: 13/02/2013; aceptado con modificaciones: 04/07/2013

RESUMEN: El delirio erotomaníaco se menciona desde la época griega. Es en el siglo XIX, con Clerambault, cuando se hace una descripción más exhaustiva de esta ideación delirante crónica de ser amado. Existen pocos estudios descriptivos y generalmente se trata de casos aislados. En este trabajo se relata de forma detallada el seguimiento prolongado de una paciente con un delirio erotomaníaco; realizando especial incidencia en la complejidad de su diagnóstico y abordaje terapéutico.

PALABRAS CLAVE: delirio erotomaníaco, delirio de Clerambault, pronóstico, tratamiento.
ABSTRACT: The erotomania delusion is mentioned from the greek era. It was in the nineteenth century, with Clerambault, when is done a more exhaustive description of this chronic delusional ideation of being loved. There are a few descriptive studies and usually these are isolated cases. In this work is recounted in detail the long follow up of a patient with erotomania delusion; making special incidence in the complexity of its diagnostic and therapeutic approach.

KEY WORDS: erotomania delusion, Clerambault delusion, prognosis, treatment.

\section{Introducción .}

El delirio erotomaníaco o de Clerambault es un subtipo de trastorno delirante en el que el tema central es la idea delirante de que una persona está enamorada del sujeto. La idea delirante suele referirse a un amor romántico idealizado, a una unión espiritual más que a una mera atracción sexual (1).

Desde la antigüedad, los delirios erotomaníacos han sido descritos con más frecuencia en mujeres. En la época griega ya se mencionaba una enfermedad de características similares (Hipócrates, Galeno), aunque es Esquirol, quien la considera como una monomanía intelectual, es decir, una alienación mental que afecta parcialmente a la mente preservando las facultades intelectuales intactas, denominándola locura del amor casto (2). Kraepelin incluye los delirios erotomaníacos en los delirios paranoicos o paranoia. Se define este proceso como un delirio lúcido, generalmente con ausencia de alucinaciones, con conservación de la actividad psíquica, con evolución extensiva de las interpretaciones delirantes, incurable pero sin evolución demencial (3). 
En el siglo XIX Clerambault describe la erotomanía como la ilusión delirante de ser amado, trazando un cuadro clínico minucioso con tres fases: esperanza, despecho y rencor (4).

El delirio erotomaníaco puede presentarse integrado en distintos procesos mentales como trastorno delirante, esquizofrénico o esquizoafectivo. En este trabajo se presenta el seguimiento durante más de 30 años de una mujer afecta de trastorno delirante erotomaníaco o delirio de Clerambault. Determinados aspectos clínicos, así como del abordaje terapéutico y el seguimiento prolongado de la paciente, ofrecen la posibilidad de realizar una reflexión sobre esta patología mental.

\section{Caso clínico}

\section{Antecedentes biográficos, médicos y psiquiátricos}

Mujer de 70 años, soltera, vive sola con supervisión constante por hermana y cuñado que viven en el piso inferior al suyo. Describen embarazo, parto y desarrollo psicomotor normales, así como una infancia feliz, buen trato con padres y hermana menor.

Mantuvo una relación estable durante 10 años, entre los 28 y 38 años, con hombre casado de situación social superior a la de ella (era su jefe en el Ministerio donde trabajaba). Mujer de carácter extrovertido, abierta, con buen apoyo familiar y social. Diplomada en Graduado Social y Humanidades, trabajó como administrativa durante 20 años en un Ministerio en Madrid tras obtener la plaza por concurso oposición. Jubilada desde 1992, año en el que se le dio la invalidez permanente por enfermedad mental incapacitante.

Antecedentes somáticos de HTA, glaucoma, hernia de hiato, fractura de tibia y peroné derechos con injerto tras accidente de tráfico con TCE y pérdida de conciencia 1981.

En seguimiento desde hace 32 años por servicios de Psiquiatría de diferentes hospitales (38 ingresos) y Unidad de Salud Mental (USM) del país (Madrid, Valencia y actualmente desde 2000 en Xàtiva) por trastorno delirante erotomaníaco de Clerambault, habiendo recibido previamente multitud de diagnósticos y tratamientos. Respecto a antecedentes familiares, una hermana con trastorno distímico y un sobrino consumidor de tóxicos con suicidio a los 20 años.

\section{Enfermedad mental y evolución}

Inicio de sus trastornos mentales alrededor de los 38 años coincidiendo con accidente de tráfico en el cual fue atropellada mientras cruzaba la calle, con el resultado de fractura de tibia y peroné derechos y TCE con pérdida de conciencia, que precisaron ingreso hospitalario en la planta de Traumatología durante varias 
semanas y cirugía reconstructiva. Mientras estuvo ingresada fue visitada con regularidad por varios de sus compañeros de trabajo, pero no por su ex-amante con lo que ella empezó a interpretar cualquier gesto de éstos como indicativo de que habían sido enviados por éste para espiarla, vigilarla y trasmitirle información de su estado, dado que él no podía asistir a verla. También empieza a notar pensamientos e ideas extrañas en su cabeza; ideas obsesivas en relación a la figura de éste, centradas en conseguir que vuelva a retomar la relación con ella.

Tras el alta hospitalaria estas ideas van aumentando en intensidad, cree verlo por doquier, no acepta ni comprende que él no sienta lo mismo por ella, derivando en conductas inapropiadas de llamadas telefónicas reiterativas, visitas a su despacho en horario laboral, increpándolo a volver a retomar la relación.... Todo ello acompañado por clínica de ansiedad intensa, insomnio, anorexia, abandono de las obligaciones laborales y de su autocuidado personal, dificultad para concentrase en las cosas, gastos de sumas elevadas de dinero en ropa y regalos para su amado, por lo que es llevada a urgencias del hospital psiquiátrico de zona donde se le ingresa y se le pauta tratamiento sedativo y psicoterapia con mejoría absoluta de la ansiedad, pero con persistencia del aparente estado delirante. En las entrevistas que se llevan a cabo durante el ingreso la paciente no presenta alteraciones de conducta ni problemas de convivencia con el resto de pacientes, reconoce que su forma de vivir la realidad le perjudica, pero no puede evitarlo, impresionando de fondo neurótico y reacciones de tipo histeroide, para hacerse de notar dentro de sus compañeros de trabajo. Recibió el alta con el diagnóstico de trastorno ansioso-depresivo versus neurosis obsesiva, pero con la duda de si no habría de fondo una psicosis delirante y pautándosele tratamiento con tioridazina, haloperidol y amitriptilina.

Tras el alta hospitalaria mantiene seguimiento periódico durante unos meses en USM de zona. En una ocasión es acompañada a urgencias psiquiátricas por un amigo presentando conductas extravagantes delante de la puerta de su centro de trabajo paseando un cochecito de bebé vacío, hablando sola y escenificando que está cuidando de su bebé imaginario. Tras ser valorada, no presenta alucinaciones, ni ideación autolítica, ni clínica delirante, haciendo crítica de lo sucedido ("lo hago para llamar al atención de mi amado") y refiriendo que no lo va a volver a hacer, se le da el alta con diagnóstico de estado crepuscular histérico ya cedido, pautándosele clorpromazina y clorazepato.

Meses después presenta un "nuevo episodio crepuscular", pero mucho más elaborado y de mayor intensidad y duración, consistente en las compras excesivas de varias bolsas con ropa infantil, comida para niños y objetos de aseo masculinos, permaneciendo durante días en un parque situado enfrente de la casa de su "amado", representando que vive en familia con él y sus 2 hijos, un niño y 1 niña. Durante esos días no acude a su trabajo, vive y pernocta en dicho parque, apenas come y casi ni duerme. La enferma a su ingreso hace crítica total de lo ocurri- 
do, se reprocha la pérdida de tiempo, de su imagen personal y económica que va a suponer para ella, relatando que es como una fuerza, una energía interna, una pulsión, la que la lleva a realizar dichos actos (hiperactividad, deseos amorosos descontrolados, con ciertos tintes hipertímicos) pero deja la duda de si existe una total ausencia de conciencia de sus actuaciones. Mantiene la convicción, delirante, bizarra, contumaz, de que su ex-amante la sigue amando a pesar de las circunstancias reales. Se plantean los posibles diagnósticos de psicosis histérica, erotomanía o psicosis psicógena, pautándosele al alta tratamiento farmacológico clorazepato y flurazepam y derivándola a USM para inicio de psicoterapia psicodinámica.

En los años siguientes a esta última alta presenta varios episodios de reagudización del cuadro anterior, algunos de ellos de menor intensidad y duración, siendo atendida en urgencias psiquiátricas, sin ingresarla, recibiendo como tratamiento clorazepato im y remitiéndose a su domicilio para proseguir su tratamiento psicoanalítico habitual.

En aquellos episodios en los cuales la intensidad es mayor, se realizan ingresos hospitalarios para controlar el comportamiento (nada más llegar al hospital hay un rápido y total apaciguamiento de las conductas pulsionales, permaneciendo la paciente en una posición de "belle indiference"), no precisando como tratamiento farmacológico nada más que benzodiazepinas durante los mismos.

En uno de estos episodios de mayor intensidad, por lo que precisa ingreso, se objetiva un ánimo depresivo sin síntomas psicóticos, durante el cual realiza fuga hospitalaria en 2 ocasiones, relatando la paciente que lo ha hecho de manera impulsiva y sin poder controlarse, no existiendo sintomatología delirante manifiesta como justificación, siendo pautado perfenacina y amitriptilina.

Dado que las recaídas son constantes con las consiguientes fugas y ausencias laborales que conllevan, se le da la invalidez laboral total y permanente, a la edad de 50 años. La paciente abandona la ciudad donde trabajaba y residía, trasladándose a vivir a su pueblo natal. Se somete a un nuevo tratamiento psicoterapéutico con un psiquiatra amigo de la familia durante un período aproximado de 10 años, en el cual presenta algunas recaídas coincidentes casi siempre con el abandono de medicación neuroléptica y antidepresiva. Abandona el tratamiento con este psiquiatra por presentar la idea delirante de que estaba enamorado de ella.

Después de diez años sin ver a su ex-amante (aunque habiendo tenido contacto telefónico con llamadas reiterativas por parte de ella) viaja en tren hasta la ciudad donde él residía con la intención de volver a verlo. Es llevada al hospital psiquiátrico por unidad SAMUR tras encontrarla intentando cruzar la autopista de varios carriles con grave riesgo para su vida. Ella relata el hecho como que es víctima de un impulso que no puede controlar y que actúa en ocasiones en contra de su voluntad. Dicho impulso va encaminado a reunirse con su amado (a pesar de saber que está casado y que no le corresponde). Refiere sentirse anímicamente 
NOTAS CLÍNICAS

deprimida por no poder controlar su "obsesión amorosa", por lo que ha intentado quitarse la vida cruzando de manera arriesgada dicha autopista. Durante el ingreso hace crítica de lo sucedido manteniendo cierta preocupación por la aparición de dichos impulsos que ella considera desagradables. Se le diagnostica de TOC y se le pauta litio, paroxetina y clorazepato.

Tras la muerte de su sobrino por suicidio es ingresada en hospital por presentar un cuadro de mutismo, anergia, deterioro cognitivo manifiesto, cierto nivel de confusión, desorientación temporo-espacial, falsos reconocimientos, pérdida de memoria inmediata y reciente, nula capacidad para la abstracción o el cálculo, lenguaje divagatorio, tangencial y plagado de pararrespuestas, sin sintomatología delirante. Se realizan varios "minimentals" con resultados contradictorios entre ellos; TAC craneal sin hallazgos patológicos; EEG para descartar foco epiléptico que resulta normal; analítica completa con hemograma, bioquímica, vitamina B12, hormonas tiroideas, serología, con resultados normales; siendo diagnosticada de pseudodemencia de aspecto histérico versus trastorno disociativo y pautándosele tratamiento al alta con litio, biperideno, risperidona y clorazepato.

A los pocos meses de este último ingreso, realiza una nueva fuga de su domicilio hacia la ciudad donde trabajaba, presentando conductas inadecuadas de compras excesivas de ropa de niños, ropa masculina, así como deambular sin rumbo y sin descanso cargada con dichas compras, poniendo en peligro de nuevo su integridad física. Al ingreso hospitalario presenta por primera vez alucinaciones auditivas de voces imperativas que ordenan y dirigen sus actos en contra de su voluntad, junto a un síndrome de influencia que acapara su voluntad, inutilizándola, siendo vivida subjetivamente como una fuerza o impulso interior que la lleva a realizar determinados actos sin que ella pueda oponerse (caminar sin control alrededor de las inmediaciones de la casa de su ex-amante porque si deja de caminar algo malo va a sucederle a ella, a su familia y poder perder sus pertenencias y su dinero). Al alta recibe diagnóstico de psicosis inespecífica y se le pauta olanzapina y clorazepato.

En los años siguientes alterna períodos intercrisis sin clínica afectiva ni psicótica, con un correcto nivel y funcionamiento cognitivo, junto a varios ingresos de similares características, probablemente desencadenados por la disminución o el abandono de la medicación, recibiendo diferentes tratamientos: clozapina, carbamazepina, valproato, quetiapina, amisulpiride, zuclopentixol depot. El período intercrítico de mayor duración en este tiempo es de 3 años, coincidiendo con la administración regular del zuclopentixol depot; apareciendo de nuevo clínica, tras la disminución de la dosis. Al ingreso realiza crítica de lo sucedido, pero no parece poder controlarse, refiriendo como si alguien le hubiera puesto una penitencia que consta de 3 pasos: ir al municipio donde trabajaba, comprar regalos para su amado y sus supuestos hijos en común y caminar sin parar. Si no cumple alguno de los 3 pasos, la penitencia no es válida y algo malo le ocurrirá a ella y a sus seres queri- 
dos. Sus familiares confirman el total abandono de la medicación (zuclopentixol depot, paroxetina, quetiapina, diazepam y lormetazepam), sintiéndose desbordados ante la situación. Se le diagnostica definitivamente de trastorno delirante erotomaniaco y se le pauta risperidona im/15 días. Permanece estable durante meses pero presenta nuevas recaídas coincidentes con la disminución de dosis de risperidona im, así como el abandono del tratamiento psicoterapéutico. Estable clínicamente, sigue control ambulatorio en USM durante 3 años. Se le cambia la medicación de risperidona $\mathrm{im} / 15$ días a paliperidona $\mathrm{im} /$ mensual porque la paciente presenta efectos secundarios. Una vez más, tras la disminución de la dosis de paliperidona (de 100 a 75 mgr), presenta una nueva reagudización clínica consistente en conductas apragmáticas, rechazo a la ingesta y tratamiento, abandono de autocuidado, llamadas telefónicas a su ex-amante, todo ello congruente con su delirio erotomaníaco. Durante el ingreso se observa una buena evolución tras la reinstauración del tratamiento parenteral, oral y psicoterapéutico. Se le da el alta y actualmente se encuentra estable, con buena adherencia a pautas terapéuticas.

\section{Discusión}

El trastorno delirante erotomaníaco o delirio de Clerambault es una enfermedad infrecuente en la población general, existiendo pocos estudios concluyentes sobre prevalencia, tratamiento, evolución y pronóstico (5-6).

La relevancia del presente caso viene dada por la exhaustiva información recogida de la paciente durante un seguimiento de 32 años, que permiten aportar datos clínicos, de tratamiento y evolución muy significativos.

Llama la atención del caso, por no ser habitual, un inicio larvado y tras varios años de finalizar una relación real; en los casos documentados por revisión bibliográfica describen pacientes que no han tenido relaciones reales con el sujeto "idealizado amorosamente" y con un inicio brusco sin ningún desencadenante (7). Su evolución ha sido hacia la cronicidad, manteniéndose en todo momento en la etapa de esperanza de conseguir su propósito de reunirse con su amado y conseguir su amor, sin presentar despecho, rencor o enfado ante las negativas de éste, como sucede en los casos descritos por Clerambault (4).

A lo largo del seguimiento en los distintos servicios de salud mental, la paciente recibe numerosos y variados diagnósticos, así como distintos abordajes terapéuticos psicofarmacológicos y psicoterapéuticos. Resulta llamativa la disparidad de diagnósticos durante los primeros años de enfermedad, pasando desde la neurosis a la psicosis. Estas discrepancias se relacionan con el tipo de delirio erotomaníaco y los diferentes modelos para su comprensión y abordaje desde la Psiquiatría. A 
pesar de su evolución crónica, no se observa deterioro cognitivo, si bien su funcionamiento personal se encuentra marcado por el delirio. Al igual que en el sueño, en el delirio existe también un cumplimiento de deseos, como si sirviera para ocultar la derrota en la vida. Esta evolución es reflejada en los casos descritos en la literatura (7-8).

Durante años las remisiones clínicas eran mínimas, realizando un cumplimiento irregular de los tratamientos psicofarmacológicos, debido a su escasa conciencia de enfermedad. De todas las intervenciones terapéuticas, se debe destacar los dos períodos de tratamiento y seguimiento con psicoterapia psicodinámica, donde se objetivaron períodos asintomáticos sin precisar ingresos hospitalarios ni descompensaciones clínicas, así como con la administración de neuroléptico depot parenteral. En la actualidad y desde hace más de tres años se encuentra más estable, sin reagudizaciones psicóticas graves (consistentes en activación de un delirio crónico erotomaníaco con planificación y fuga para reunirse con su amado). Las veces que se ha intentado la reducción de la dosis del mismo, a petición de la paciente, ha presentado leves descompensaciones que han sido minimizadas mediante ingreso en Unidad de Hospitalización Psiquiátrica para reintroducir la dosis habitual, consiguiendo la estabilidad clínica.

En la bibliográfica existen pocas referencias respecto al tratamiento de esta entidad (9-11), precisando de estudios con más casos para poder avanzar en el abordaje de este apasionante trastorno mental.

\section{BIBLIOGRAFÍA:}

(1) American Psychiatric Association. Diagnostic and Statistical Manual of Mental Disorders, DSM-IV-TR, 4th edn. Washington, DC: APA, 2000.

(2) Esquirol, JED.: "Memorias sobre las locuras y sus variedades"; De. Dorsa, Madrid, (monomanía erótica)- Traducción del libro "Des maladies considerées sous les rapports medical, hygieniques et médico-legales" (1991) pp. 315-326.

(3) Kraeplin E: Maniac Depressive Insanity and Paranoia. Edinburgh, Robertson, Lvingstone, $1963, \mathrm{p} 245$.

(4) Clerambault GG. El automatismo mental. Madrid, Dor, 1995.

(5) Kelly Brendan D. Erotomania epidemiology and management. CNS Drugs 2005; 19: 657-669.

(6) Berrios GE, Kennedy N.Erotomania: a conceptual history. Hist Psychiatry 2002; 13(52):381400.

(7) Jordan HW, Lockert EW, Johnson-Warren M, Cabell C, Cooke T, Greer W, Howe G. Erotomania revisited: thirty-four years later. J Natl Med Assoc 2006; 98(5): 787-93.

(8) Jordan HW, Howe G. De Clerambault syndrome (erotomania): a review and case presentation. J Natl Med Assoc 1980; 72 (10): 979-85. 
(9) García-Fernández L, Alcántara López AG, Martínez Herrera MJ. Paranoia erotica (Clerambault syndrome) successfully treated with risperidone. Ann Pharmacother 2002; 36 (12): 1972-3.

(10) Alao AO, Armenta W, Yolles JC. De Clerambault syndrome successfully treated with olanzapine. Ann Pharmacother 2000; 34 (2): 266-9.

(11) Vrieze E, Pieters G. De Clerambault syndrome. A case report and a review of the literature on the difference between primary and secondary erotomania. Tijdschr Psychiatr 2007; 49 (11): 845-9. 